Fecha de recepción: febrero 2019

Fecha de aceptación: julio 2019

Versión final: octubre 2020

\section{La investigación de aspectos sociales y culturales como estrategia de Diseño}

Marco Antonio Sandoval Valle *

Resumen: Pareciera indiscutible que como parte de la estrategia en la investigación y producción de Diseño se impliquen aspectos sociales y culturales, en la realidad esto es algo que no ocurre necesariamente, ni en lo formativo ni en lo profesional, pues constantemente se aprecian inconsistencias conceptuales y formales, motivadas por presiones económicas, limitaciones profesionales y exigencias de todo tipo, aunque fundamentalmente definidas por una visón paradigmática que define los alcances de la investigación y de la realización de Diseño. A continuación se presenta una caracterización general de la relación que se estima guarda la investigación en Diseño con respecto al campo profesional, al considerar la realización de proyectos; la concientización de un método al momento de producir y el proceso de registro para la socialización de lo que se hace. También se pone énfasis en la descripción de la investigación desde los sectores del Diseño como son el académico y el del medio laboral. Se procura ponderar al Diseño desde una perspectiva social, ya que los individuos son la razón fundamental de lo que se planifica y produce. $\mathrm{Al}$ pensar oportunamente a la cultura y al orden social en proyectos e investigaciones de Diseño, se advierte la inseparable incidencia de ambas en cualquier desarrollo estratégico, determinándose también las repercusiones de su eficacia. Se señalan tres momentos conceptuales respecto al diseñar e investigar: su condición social; su condición externa y su desustancialización. Éstos momentos se abordan a partir de fundamentos sociológicos con el fin de ser consistentes con los objetivos de la exposición, al procurar concientizar y revalorar los aspectos sociales de los procesos al hacer e investigar disciplinariamente, y así procurar poner al Diseño en el debate social y cultural al ratificarlo como profesión dinámica que proviene de rasgos colectivos, que contribuye a configurar pensamientos e incidir en comportamientos.

Palabras clave: Investigación en Diseño - Diseño social - investigar - diseñar.

[Resúmenes en inglés y portugués en las páginas 99-100]

(*) Profesor Titular "A" de Tiempo Completo en el Posgrado de Artes y Diseño en la Facultad de Artes y Diseño (FAD) de la UNAM, sus investigaciones y cátedras están relacionados con temáticas de dibujo, Diseño, imagen, cultura y lenguaje. Es doctor en Artes y Diseño por la misma Universidad. 


\section{El entorno de la investigación en el Diseño}

Como se ha mencionado, este texto procura concientizar respecto a la importancia que tienen las personas al momento de investigar y planificar Diseño. Cabe destacar, lo difícil que resulta aproximarse a los temas de investigación sin reflexionar en la influencia que tienen en el desarrollo de productos investigativos los centros, institutos y universidades en las que se enseña el Diseño. En condiciones usuales se vincula la investigación al contexto de las ciencias, la educación o la cultura, específicamente con las ciencias duras o las ciencias sociales, e incluso las humanidades; sin embargo, en la percepción cotidiana difícilmente se relaciona al Diseño, y menos aún con el gráfico o de la comunicación visual -probablemente el industrial y el arquitectónico han tenido otro desarrollo.

Incluso puede observarse que para las perspectivas de las políticas públicas de educación y de investigación, en el caso de México y desde el Consejo Nacional de Ciencia y Tecnología CONACYT, las disciplinas del Diseño -así como muchas otras- son consideradas profesionalizantes y no de investigación, es decir tienen una orientación profesional y responden a necesidades específicas de sectores concretos de la sociedad. Es en este escenario que se capacitan individuos para el ejercicio de la profesión y no de la investigación, por su sentido eminentemente práctico - pues son pensadas para la resolución de problemas-. Se padece de este señalamiento más allá del tipo de Diseño que se enseñe -gráfico, de comunicación visual, industrial, constructivo, de interiores, entre otros- desde la máxima autoridad política y gubernamental; la cual define en dónde, cómo y en qué condiciones se da la investigación y donde no. El responsable de trazar los límites de lo que es ciencia o tecnología atenta desde el origen en la estructura misma de la conformación disciplinaria del Diseño, pues limita sus alcances en todos los sentidos, -no solo los de la investigación-. Esta determinación no es cosa menor, ya que trastoca múltiples esferas de trascendencia, de lo que se hace y cómo se hace en los campos de estudio, en su aplicación profesional, e incluso repercute en la remuneración económica. Así, resulta una tendencia el repensar sobre todo desde la academia- la investigación en Diseño con el fin de validar y dar cuenta del conocimiento que implica y se produce cuando se diseña; y por otra parte, desde la autoridad científica, existe resistencia o se ignora que al diseñar se desarrolla cierto tipo de actividades ligadas a otras formas de investigación.

Bernardo Lahire (2006) realiza un razonamiento para la sociología perfectamente pertinente para el Diseño: constantemente se está transitando en saber cuál es la "razón de ser" de la profesión, hay un evidente interés, por lo menos en quienes le otorgan una utilidad, en explicar su razón de ser. En el caso del Diseño los mayores posicionamientos sobre la necesidad de investigar provienen de la academia, y en cuanto a su importancia social desde el propio gremio. Menciona Lahire (2006) que "los diversos oficios que componen la formación social están desigualmente justificados en su existencia y desigualmente trabajados por la cuestión de su utilidad social" (p. 22).

En México ha sido notable una creciente teorización del Diseño en general, y del de la comunicación visual en particular, a partir de la formalización de los procesos de investigación, situación que a finales de la década de los años 90 y principios del 2000 no era un escenario común ni constante, ya que las principales aportaciones teóricas a la disciplina del Diseño eran ofertadas desde actores con formación en áreas no afines, pero que por 
ciertas circunstancias habían llegado a desarrollar laboralmente algún proceso de Diseño o a insertarse en los espacios académicos de alguna área intra-disciplinaria no cubierta por los diseñadores, principalmente en programas emergentes.

Por consiguiente, se motivaron participaciones interdisciplinarias de antropólogos, sociólogos, psicólogos, filósofos, pedagogos, historiadores, lingüistas, y por supuesto, arquitectos, diseñadores industriales, historiadores del arte, entre otros; sin embargo había pendientes gremiales ya que la participación y análisis a través de medios escritos no eran abundantes y mucho menos realizadas por los propios diseñadores.

La percepción y producción de los diseños han sido constantemente resignificadas a consecuencia de los usos, funciones y contactos culturales con los que se ven expuestos en diferentes planos de acción, tales como: espacios académicos, despachos, agencias, talleres de producción, espacios de difusión cultural, sectores económicos en general y, por supuesto, el propio entorno social. Desde la teoría de las profesiones Eliot Freidson (2001) acota la naturaleza de la profesión desde una "concepción genérica de las ocupaciones. Asimismo, aborda el concepto como una construcción histórica de ciertas sociedades, y analiza de manera general el desarrollo, uso y consecuencias que tal concepto tiene en esas sociedades" (p. 28).

Como se comentó, no es común encontrarse con el concepto de investigación en el contexto de los diseños, sin embargo es cada vez mayor la exigencia en los ámbitos universitarios de realizarla. Resulta necesario tener publicaciones, papers, artículos, libros, entre otros, para ser considerados en el reconocimiento y posicionamiento de un investigador-diseñador con seriedad. Pareciera que existe una encrucijada indudable de demandas sobre lo que se debe hacer en el Diseño para ser identificados en los entornos de la investigación, como si la contribución a la vida objetual, conceptual, económica y estética que acompaña a las sociedades no ratificara la razón de ser del Diseño. Sin embargo la investigación resulta necesaria y urgente.

[...] por un lado, con una disciplina académica y científicamente menos legítima que otras (por ejemplo, la física, la química, las matemáticas las neurociencias, etc. )... el sentimiento de utilidad o inutilidad de un saber a menudo no proviene tanto de la índole de ese saber como de su valor académico y extra académico (débil o fuerte prestigio de los estudios, pocas o muchas salidas profesionales, pequeñas o grandes reputaciones de los empleos ocupados). La alta legitimidad y el gran valor (económico y simbólico) que el mundo social atribuya a ciertas actividades salen al paso de toda interrogación medianamente importante sobre sus razones y su utilidad (Lahire, 2006, p. 24).

Lo que constantemente es notable de los diseños son sus productos -aunque ahora también experiencias de usuario- es decir, muchas veces se aprecian sus consecuencias en cuanto a su utilidad, procesos comúnmente objetuales o acciones que provienen de un ejercicio conceptual. De tal manera que se mira sólo una pequeña parte de la totalidad que implica la actividad de diseñar -pensamiento conceptual y técnica-, condición que facilita que el público externo se vea a sí mismo en una posición de encantamiento o desprecio desde el uso, gusto, o necesidad, sobresimplificando el pensar y hacer de las especialidades 
de la disciplina. Esto conlleva de forma tácita que la concepción de la actividad de diseñar fuera producto de un acto de inspiración, ocurrencia o arbitrariedad individual; condición que propicia una falta de valoración de la importancia, responsabilidad, sofisticación y rigor que deberían ser propios de la profesión y que estas disciplinas requieren al momento de producir e implicar los propios procesos de investigación.

El fenómeno de la profesionalización ha impactado en escuelas, centro educativos o institutos, más allá de si tienen una orientación paradigmática, "científica, artística, social, empresarial”, o si su propuesta de formación en el Diseño apelan a lo funcional, estético, al proyecto o al plan de negocio. Se puede relacionar este fenómeno por una prioritaria producción de proyectos u objetos. Desde las profesiones Flora M. Hillert (2009) menciona:

Las profesiones se definen en función de un acampo de saberes teóricos y prácticos especializados que son utilizados o demandados por la sociedad, cada profesión supone un conjunto de trabajadores intelectuales que se ha formado y perfeccionado para desarrollarlos y transferirlos a través de un extenso proceso de aprendizaje que acredita la posesión de ciertos saberes y aptitudes (p. 2).

Al margen de su carácter, los diseños han compartido saberes y prácticas importantes, lo que permite un intercambio sustantivo que le da sentido al desarrollo investigativo. López Cano (2015) desde la perspectiva de la investigación-creación, presenta una serie de elementos a considerar en áreas de trabajo prácticas, con tipos de conocimiento y problemas de implementación particulares. Aunque su reflexión parte de la creación musical, se extiende al Diseño al considerar ciertas coincidencias conceptuales y profesionales; como su condición de expresar una serie de conocimientos basados en la práctica, así como ambas compartir el ser disciplinas creativas.

Cano menciona que en la investigación-creación aún no hay consensos sobre su definición; desde los centros de enseñanza o desde los órganos oficiales no hay políticas o métodos de investigación que estén perfectamente delineados y que le determinen, indefinición que se extiende a las maneras y formas propias de reportar resultados. Se considera que esta situación afecta también al Diseño.

El autor caracteriza tres aproximaciones a la investigación. En una primera instancia presenta los discursos homologadores, centrado en el reconocimiento de que en la investigación se produce conocimiento a consecuencia del rigor de búsqueda y de reflexión respecto a la información obtenida al momento de diseñar. Se parte de que el Diseño genera una experiencia de conocimiento -sin embargo, sin definir cómo, en qué condiciones y en forma de qué productos se genera-. Hay una suerte de consenso común, en la generación de conocimiento en el proceso de Diseño, aun sin precisar sus formas; por lo que en las instituciones ha habido una exigencia de homologar la producción de Diseño como actividad con la investigación científica. La idea es, validar los objetos resultantes del quehacer del diseñador, cuando se hace un proyecto particular de editorial, arquitectónico, objetual, de modas o textil. Es decir, empieza a existir la solicitud de que estos productos o proyectos sean aceptados igualmente en las evaluaciones universitarias, sobre todo para razonar la productividad de los académicos, sin embargo no hay lineamentos ni metodologías precisas para su realización, se navega en una suerte de nebulosa sobre su aplicación. 
Por otra parte, hay una especie de "diseñador-investigador", el cual se esfuerza por acceder al reconocimiento en el ámbito intelectual de la ciencia y, en un sentido más práctico, al acceso a apoyos de orden económico, becas, y a la participación en congresos y redes de investigación; por lo que resulta necesario recurrir a la producción científica habitual: la realización de artículos, libros o textos en general. La idea de realizar investigación en la práctica diseñística con respecto a las áreas científicas, trae consigo varios riesgos en el individuo o las instituciones, pues en algunos casos, existe una exigencia laboral doble al quehacer profesional -sobre todo en el ámbito académico-. En muchos casos se cambia la razón esencial de la profesión formativa; el diseñador forzado con sus características y metodologías a cuadrar dentro de los cánones de la investigación de las ciencias duras, o dicho de otra manera, que se pondere el trabajo de la de la investigación científica por encima de la naturaleza y metodologías del trabajo del propio Diseño. Esto ocurre en otras áreas profesionalizantes, por ejemplo pensar que un ingeniero para validar su trabajo en investigación debe dejar de lado lo propio al ejercicio de su profesión -construir presas, carreteras, puentes- para escribir artículos.

En un segundo momento López Cano habla de los discursos institucionales hegemónicos, que son descritos como aquellos espacios o instituciones que han ganado experiencia y formalidad en la investigación específicamente -en este caso el Diseño-. Estos se caracterizan por una amplia actividad a través de la producción de artículos, congresos, cursos, libros, etc., de tal forma que estos centros llegan a ser un arquetipo para individuos e instituciones que trabajan en ese campo de conocimiento, sin embargo tampoco han clarificado y precisado del todo, cómo son los procesos y metodologías de sus procesos de investigación.

Aunque es clara la reflexión que hace Cano aplicada al ámbito de la investigación artística, en la aplicación hacia los fenómenos del Diseño existe una importante paridad, puesto que advierten dos momentos fundamentales, la investigación en Diseño no puede ocurrir separada de la producción, y por otra parte sin la investigación en los ámbitos académicos. Eguzi Urteaga (2008) en la sociología de las profesiones advierte la "crisis como consecuencia del abismo que separa la realidad profesional de su representación intelectual. Las teorías, los conceptos y los métodos propuestos son cada día menos apropiados para comprender las profesiones en su complejidad" (p. 169). Por lo cual, es necesario el reconocimiento de la mutua relación y trabajo continuo, en aras de profundizar en las condiciones de utilidad e investigación del Diseño.

Se entiende que reflexión teórica y producción son necesarios e importantes en el desarrollo de la investigación, y también obligados, ya que, al final, lo que otorga el carácter a un producto de investigación será la generación de conocimiento indispensable a la comunidad que lo requiere, en ese caso existen dos momentos relevantes, la manera en que se documenta lo que se investiga y como se socializa; es decir cómo se construye un discurso con base en la producción.

Es común que en el gremio del Diseño haya fragmentación en relación a lo que ocurre entre el medio laboral con respecto a la academia. Por una parte, desde la producción de Diseño en el ámbito profesional se señala que en las instituciones hay un profundo desconocimiento de lo que se vive en la cotidianeidad de los centros de trabajo, puntualizando en que, en muchos sentidos, la institución es totalmente ajena al despliegue tecnológico y 
a la actualización profesional; por parte de la academia se considera que es desde allí que se producen reflexiones teóricas e investigativas trascendentales que redimensionan lo que se hace en el Diseño profesionalmente. Sin embargo no se tiene en cuenta que esta condición en la realidad de la práctica del Diseño no encuentra posibilidades de implementación a consecuencia de las jornadas extenuantes y, en muchos casos, los salarios castigados que imposibilitan el sentido reflexivo de lo que se hace,

En relación con los saberes, cada grupo posee saberes compartidos con el otro y saberes específicos. La división de posiciones y saberes se concreta entre los sujetos; por lo mismo, cada sujeto ocupa ciertas ubicaciones y pone en juego ciertos saberes. Estos saberes, producidos en un contexto histórico, hacen referencia a sus respectivas culturas, expresan modos de socialización y posicionamientos ante la realidad (Hillert, 2009, p. 1).

La compartición y diferenciación entre las experiencias y conocimientos en la realidad pone en conflicto las dimensiones de hacer e investigar Diseño, situación que contribuye también a generar cierto carácter identitario, por una parte diferenciador y por otra coincidente. Hillert lo expresa como la identidad profesional de un grupo; las coincidencias se dan en el manejo de ciertos campos semánticos tanto en la experiencia profesional como en la académica -en cuestiones metodológicas, discursivas, compositivas, perceptuales, teóricas, objetuales, tecnológicas, económicas, entre otras- sin embargo, también se ponderan paradigmáticamente unas cuestiones más que otras, lo que lleva al conflicto de lo que ocurre disciplinariamente.

En un tercer momento López Cano (2015) presenta su llamada propuesta específica, en la cual trata de organizar diferentes herramientas de orden conceptual y metodológico que permitan colocar a una comunidad interesada en los aspectos investigativos a través de direccionar la reflexión y elaboración de discursos, sin dejar de lado la producción de Diseño; esto implicaría no solo elaborar discursos teóricos sino también redundar en propuestas productivas en torno al mismo, que de alguna manera incidan y transformen el entorno profesional.

En una primera instancia, será importante reflexionar respecto a la investigación productiva en el Diseño, a partir llevar a cabo prácticas de realización que incluyan elementos técnicos y tecnológicos fundamentadas en sus productores, para incorporar rasgos particulares y estilísticos de esas personalidades y sus circunstancias específicas a la investigación de Diseño, un conocimiento orientado al hacer. En otras palabras, teorizar y experimentar a partir de elementos prácticos sobre los procesos de realización.

En otro nivel se habla del proceso creativo. Dadas las complicaciones semánticas es adecuado plantearlo en términos de concientización del proceso; no dejar de lado los conocimientos de cómo se construyen los proyectos, atendiendo la realización del Diseño. En este sentido va a ser muy importante cómo se documenta el proceso de creación, fundamental en la elaboración de reportes y descripciones. En un tercer nivel se hablaría de reflexionar y analizar el ejercicio profesional aludiendo a cómo ocurre el Diseño en tiempo y espacio, la experimentación en el entorno para atender una problematización. 
Por último aborda las técnicas personalizadas que se ponen en práctica, como método y técnica en la realización de un objeto de Diseño o de un proyecto, dando importancia a las adecuaciones individuales que se hace ante cada propuesta.

En otro sentido no menos importante es necesario atender al formar para la investigación, esto es, la necesidad que hay desde el ámbito de la academia de influir en la formación del individuo para la generación de prácticas reflexivas, de modo que la investigación en las tareas cotidianas sea parte de una respuesta normalizada, es decir que haya una búsqueda de información -que en teoría debiera suceder-. La aplicación de diferentes metodologías de investigación documental, además de los métodos de elaboración proyectos, sean cuantitativas o cualitativas, sin negar que son actividades propias de la formación.

Desde esta perspectiva, se esperaría observar a un individuo que es mucho más crítico, con una formación completa, de tal manera, que se implicaran tanto los lenguajes propios del Diseño como los que permiten hacer un registro -a través de la escritura o de cualquier otro-, sin desdeñar la importancia de éstas actividades que han estado lejos del actuar profesional y académico.

Es recomendable, con un sentido autocrítico, trascender la justificación de que la investigación en el Diseño se da como extensión natural a las propias búsquedas expresivas, estilísticas y formales que se hacen; puesto que ante la transformación social y las exigencias profesionales, es importante contribuir a la conformación de masa crítica que otorgue equilibrios, por una parte sin sucumbir ante el paradigma de que las aportaciones teóricas sean las exclusivas para valorar la productividad en el Diseño, y por otra parte, tampoco es recomendable el ser determinados con criterios cuantificables de otras áreas, ya que han formulado directrices de investigación bajo sus propias necesidades.

Considerar que el Diseño Gráfico ya no puede ser valorado solo por sus aspectos formales o técnicos sino también por sus relaciones humanas. Como actividad inherente circunscrita a la interpretación de la vida social a través de hechos comunicativos, su espectro de estudio ha de desplazarse del signo a la acción, de la gramática de las formas a la movilización de los acuerdos sociales... tendría que afrontar este escenario con una revisión conceptual que permitiera salir de las fronteras en las que generalmente se mueve... exaltación del objeto, de la composición o de la forma, y esta debilidad conceptual no le permite proyectarse fuera de sus propios límites (Tapia, 2004, p. 11-12).

\section{Condición social de investigar y diseñar}

A menudo, al escuchar el termino social se anticipan preconceptos relacionados con el individuo; el individuo en relación a un grupo y/o el entorno; el comportamiento de los individuos, las relaciones humanas. Muy a menudo en el entorno del Diseño, se habla de lo social en relación con acciones o productos de índole filantrópica, sustentable, funcional, de mejoramiento de la calidad de vida de las personas etc. Los resultados de un proceso de Diseño se concretan a través de una serie de fenómenos: representativos, perceptuales, 
imaginativos, comunicativos, constructivos, mercadológicos, económicos, siempre de índole social y cultural, pues el Diseño es posible solo en relación a los otros.

En esta sección del trabajo se procurará ponderar la importancia que tiene considerar los aspectos sociales y culturales tanto para la investigación en Diseño y su realización, pues se parte de la premisa de que siempre se trabaja en relación al otro, esto es, un sujeto -o sujetos- al otro lado en el proceso. Es difícil pensar la actividad de diseñar sin deferencia hacia el contexto colectivo; conscientes o no en el proceso de Diseño hay alguien casi de manera natural que es destinatario. Dicho de otro modo, se pretende capitalizar y concientizar el quehacer del Diseño como disciplina social en la investigación, ya que es algo de lo que no se pude eximir y qué mejor que ratificarlo como estrategia.

Como se puede ver, considerar al otro es parte fundamental de la elaboración discursiva en el Diseño, puesto que es fundamental el pretexto humano para desarrollar proyectos de Diseño. Con diferentes acepciones, se ha referido a esa figura como: destinatario, aquella persona o entidad a quien va destinado un objeto o proyecto; receptor, quien recibe un mensaje sobre todo en el contexto de los procesos comunicativos; usuario, desde la perspectiva de la función de lo diseñado ligado a la necesidad de quien usa algo; cliente, desde la condición económica, quien atiende a las aspiraciones de comitente ante objetivos de carácter primordialmente comerciales; y, en la actualidad es cada vez más común hablar de producir experiencias en los individuos, dado que los intereses se han movido hacia la satisfacción de otro tipo de inquietudes y deseos, incluso de orden exclusivamente abstractos. El Diseño es una herramienta definitivamente cultural que requiere de un entorno para existir, y que también está en continua transformación, es afectado de manera dinámica por los contextos en los que se desarrolla, por lo cual es arriesgado determinar su definición. En México, por ejemplo, en lo que se refiere a aspectos culturales y sociales es diferente lo que ocurre en el norte en relación al sur, centro, oriente u occidente del país; la aproximación cultural y productiva disciplinaria del Diseño en cada región va a tener muchas coincidencias, pero también diferencias.

Esto puede contribuir a explicar la distinción de Diseño como actividad y profesión; por una lado existen muchas actividades que resultan comunes o propias para identificar lo que se hace en Diseño, en referencia principalmente a un sentido histórico, es decir por ejemplo, dibujar el pasaje de alguna novela, representar un objeto sobre un soporte bidimensional, proyectar una máquina, realizar una letra, construir una prótesis, reproducir un panfleto; desde una condición actual se puede mencionar que todas esas acciones son señaladas como relacionadas al Diseño, sin ser necesariamente el estado actual de una sola concepción semántica del Diseño, a consecuencia del continuo desarrollo en el que se encuentra.

En otro sentido, se refiere al fenómeno creciente de profesionalización que ha experimentado el Diseño, entre otras formas, a través de los estudios universitarios, que han promovido su validación como profesión, por tanto el Diseño ha visto la institucionalización en algunas de las antes ejemplificadas actividades -representativas, culturales, económicas, políticas etc.- que le dan carácter y que según el ámbito formativo de cada región y centro educativo se ha asumido con igualdades y diferencias. Por tanto la enseñanza-aprendizaje del Diseño experimenta diferentes misiones, experiencias y perspectivas, entre las cuales se encuentra su aproximación a la investigación. 
Más allá de si la escuela, centro o instituto ha asumido una orientación científica, artística y/o de negocios en su propuesta didáctica del Diseño, ya sea gráfico, industrial, constructivo, de interiores, etc., o si el profesional se ha desempeñado en unas u otras actividades, se cree que repensar la investigación social y cultural como estrategia puede dar a la creación un valor único para desempeñarse competentemente, que implica ajustes a un conjunto de diferentes actividades, así la esencia de un posicionamiento estratégico es realizar funciones diferentes que se pueden preservarse en el tiempo (Porter, 1996).

\section{Condición externa y relacional de investigar y diseñar}

Una atención constante en lo social, posibilita dar otro valor a la investigación y lograr mayor alcance con las reflexiones teóricas y prácticas, al ofrecer un factor de trascendencia a lo que se hace-observar, estudiar, investigar, cuestionar al mundo en el que se vive, pues no se puede ser ajeno a la realidad-. Mijaíl Bajtin (2000) en su aproximación a la cultura, aborda el intercambio cultural y la importancia del hallarse afuera -exotopía- para la comprensión de la cultura,

[...] la verdadera apariencia de uno puede ser vista tan solo por otras personas, gracias a su exotopía espacial y gracias a que sean otros [...] En el ámbito de la cultura, la exotopía es el mecanismo más poderoso de la comprensión. Una cultura ajena se descubre más plena y profundamente solo a los ojos de otra cultura; pero tampoco en toda su plenitud, porque llegarán otras culturas que verán y comprenderán y verán aún más (p. 159).

Como lo menciona Batjín, la profundidad de sentido será alcanzada solamente cuando se encuentra con otro sentido, en consecuencia se puede entender que la investigación y producción de Diseño serán plenos cuando se encuentren con un sentido que es ajeno; "que supera el carácter cerrado y unilateral de ambos sentidos, de ambas culturas" (Bajtin, 2000, p. 158). En otras palabras, consecuentar los cuestionamientos de la cultura ajena, del otro. Procurar trascender los modos de actuar ya asumidos, que repiten las predisposiciones del hábito y que no consideran con profundidad indagar, pensar y diseñar desde la exotopía, al plantear preguntas y respuestas redimensionando el sentido. Enriquecer un proceso dialógico, de tal manera que se entienda al otro.

En la defensa de la sociología, Lahire (2016) posiciona a los investigadores sociales desmitificando las aseveraciones de la exacerbada individualidad, según la cual se actúa y piensa de manera independiente. El autor expone que la sociología es relacional -lo que se extendería al Diseño- ya que "Uno de los grandes avances de las ciencias del mundo social en general, y de la sociología en particular, consiste en pensar de modo relacional lo que la percepción precientífica del mundo suele llevar a pensar de modo sustancial” (p. 73). En una consideración común, las personas u organismos perciben los fenómenos como divergentes e independientes; sin embargo la realidad no es autónoma ni independiente, por tanto, lo mismo en el caso de las investigaciones y en el propio Diseño no se pueden 
entender más que en una condición relacional, en relación a una serie de elementos y condiciones que se adecuan en un entorno material y simbólico vez tras vez.

Por tanto, la expresión hallarse afuera implica estar presentes en consideración de las relaciones que motivan la interdependencia del diseñar, sustentado por las preguntas de investigación que atienden al encuentro cultural. Pues, solo se puede entender al individuo en relación a otros dentro de un contexto colectivo, y las decisiones sirven a esta estructura de carácter relacional.

\section{Desustancializar investigar y diseñar}

Desustancializar y desencializar son términos que se utilizan para decir que nada es esencialmente de una manera, o que nada tiene sustancia de manera inherente. Por el contrario, se puede decir que todo depende de situaciones relacionales y que todo es en razón de diferentes causas y motivaciones. La aproximación sociológica afirma que la investigación social contribuye a desencializar los fenómenos y los individuos, aunque es un principio previsto para esta disciplina, en el ejercicio que se viene realizando y al Diseño también se aplica. En la teoría esta propuesta suena consistente, sin embargo, en la relación de las personas y en su vinculación con los eventos, a menudo se esencializan muchos fenómenos cotidianos.

Para entender este concepto proveniente de la sociología, se retoma la reflexión de Bernard Lahire (2006)

La sociología permite recobrar el poder sobre una realidad que se impone como una evidencia difícil de cuestionar. La realidad tal como se presenta ante nosotros, suele esconder las elecciones -entre otras posibles- que subyacen a ella; impide pensar en las múltiples realidades alternativas - posibles o virtuales- que se descartan constantemente (p. 68).

En el texto, en defensa de la sociología Lahire, este autor explica cuáles son y menciona que ésta otorga conocimientos fundamentales sobre diversos temas que han sido objeto del diálogo público, ya que "historiza los estados de hechos que se suponen naturales" y ejemplifica cuestiones de género, migratorias, profesionales, familiares etc. En palabras del autor citado:

También desencializa, o desustancializa a los individuos, que llegaron a convertirse en lo que son por su relación con toda una serie de individuos, grupos e instituciones... Compara y explica las transformaciones de fenómenos considerados eternos o invariantes ... Y sobre todo contradice en cada caso las mentiras voluntarias o involuntarias sobre el estado de lo real y desarma los discursos ilusorios (Lahire, 2006, p. 66).

Se considera que al hacer Diseño se han substancializado una serie de ideas, conceptos y haceres, ya que se responden de manera normalizada a cuestiones de carácter tecno- 
lógicas, artísticas o económicas. También de manera corriente se cree que el Diseño es esencialmente creativo; o inherentemente funcional; que resuelve problemáticas; que es indispensable. Es cierto que el Diseño puede ser alguna o varias de las anteriores, sin embargo no implica que en la práctica necesariamente suceda, por lo que obliga a una actitud más reflexiva en relación a la consideración paradigmática que se normaliza.

En el caso de la investigación en Diseño también es importante ser críticos, para llevar a cabo una desustancialización en cuanto a la idea de que con la exclusiva acción de hacer Diseño se está investigando. En un sentido amplio y decisivo si de esta manera fuera, no solo se tendría una aportación ya importante a la cultura material de la sociedad sino que también se habrían generado una importante cantidad de reflexiones y debates sobre el quehacer del diseñador, con mayor injerencia en el diálogo social y cultural en la actualidad, algo que no sucede. También sería relevante desnormalizar la concepción de que los únicos investigadores en Diseño son aquellos que reportan sus trabajos de manera escrita en esquemas eficientistas distantes de la realidad de la profesión.

\section{Ideas finales}

Algo fundamental para este artículo fue abordar la valoración de aspectos sociales y culturales en la práctica del Diseño como estrategias tanto para la resolución de problemas como para el establecimiento de principios de investigación, partiendo de algo muy simple, la naturaleza intrínseca de lo que se hace al estudiar y practicar profesionalmente el Diseño, al reparar en su condición como disciplina en la que se trata con los demás. Se piensa y hace para el otro, no se habla del si mismo en primer plano. Si se entiende al otro y lo otro, por lo tanto se puede ser más asertivo con los objetivos incluso no solo profesionales. En el uso y las prácticas cotidianas, es relevante pensar la cultura y el orden social en proyectos e investigaciones de Diseño, pues se advierte la inseparable incidencia de ambas en cualquier desarrollo estratégico y se determina también el alcance de su eficacia en la configuración de imaginarios y en la experiencia simbólica de las personas, ya que se determina el sentido de la vida social, debido a que es una parte fundamental del proceso de socialización.

Por tanto no es un proyecto menor el tratar de contribuir teniendo en cuenta el factor cultural y social de hacer y pensar Diseño a partir de sus modos de investigar. En este sentido Alejandro Tapia (2004) es muy claro sobre los alcances que se tienen en el Diseño, al reflexionar respecto a:

...la falta de estudios especializados que incorporen criterios de análisis y de crítica para comprender el papel que los objetos artificiales juegan en el entorno, y ante el embate de todo tipo de productos que planificados o no, caen en sus fronteras, el Diseño parece haberse marginado del debate social y cultural de la era contemporánea. A pesar del creciente flujo transformador que éste ejerce sobre el espacio social, las reflexiones teóricas sobre la cultura, la tecnología, el desarrollo social o la comunicación parecen no considerar al Diseño como uno de los fenómenos centrales de la vida contemporánea (p. 11). 
La paradoja del Diseño es que a pesar de su importante incidencia en la vida de los sujetos y al no poderlo desligar de la realidad, no figura, como menciona Tapia, en las aportaciones importantes en los análisis del pensamiento y del orden social. Esto obliga a formular búsquedas para encontrar respuestas constantes entre la investigación en Diseño, su producción y sus registros investigativos; de tal manera que en el ejercicio del Diseño exista una complicidad teórico-práctica propia de su función y utilidad que legitime su valor, tanto en el orden económico como en el simbólico.

Todo lo anterior apuntala la prospectiva de que un diseñador adquiera cierto nivel de madurez y crítica, de tal forma que sea capaz de atender los problemas de su profesión, sin dejar de lado la oportunidad de concretar los momentos en que se hace investigación. Por lo tanto, va a ser importante ponderar el lenguaje escrito en la documentación, además de los propósitos de los propios procesos de Diseño y su producción, adecuando fundamentaciones, justificaciones e interpretaciones, incorporando el valor social y cultural de manera consciente al hacer.

En síntesis, pensar el factor social en el Diseño para desustancializar las creencias, e incorporar el aspecto relacional a los fenómenos, podría entonces contribuir a tener una actitud más crítica, tomando en serio la investigación académica y/o profesional que se hace disciplinariamente de forma cotidiana.

\section{Lista de Referencias Bibliográficas}

Bajtin, M. (2000). Yo también soy (Fragmentos sobre el otro). México: Taurus, Alfaguara.

Freidson, E. (2001). La teoría de las profesiones. Estado del arte. Perfiles Educativos, 23(93), 28-43.

Hillert. F. M. (2009). Sujetos sociales del campo profesional de la educación. Representaciones mutuas de los docentes y los especialistas en el contexto de las reformas educativas. Anuario 2008 del Instituto de Investigaciones en Ciencias de la Educación. Buenos Aires, Argentina: Editorial de la Facultad de Filosofía y Letras Universidad de Buenos Aires.

Lahire, B. (2006). ¿Para qué sirve la sociología? Argentina: Siglo XXI.

Lahire, B. (2016). En defensa de la sociología: Contra el mito de que los sociólogos son unos charlatanes, justifican a los delincuentes y distorsionan la realidad. Argentina: Siglo XXI.

López, C. (2015). Educar para la investigación-creación: áreas de trabajo, tipos de conocimiento y problemas de implementación. A Contratiempo. Recuperado de http://www.musigrafia.org/acontratiempo/?ediciones/revista-25/artculos/educar-para-la-investigacin -creacin-reas-de-trabajo-tipos-de-conocimiento-y-problemas-de-implementac.html.

Porter, M. (1996). What is Strategy? Harvard Business Review, noviembre-diciembre. Recuperado de: https://hbr.org/1996/11/what-is-strategy

Tapia, A. (2004). El Diseño Gráfico en el espacio social. México: Designio.

Urteaga, E. (2008). Sociología de las profesiones: una teoría de la complejidad. Lan Harremanak 18(2008-I), 169-198. Recuperado de www.ehu.eus/ojs/index.php/Lan_Harremanak/ article/download/2812/2428 


\begin{abstract}
It might indisputable for social and cultural aspects to be involved within design's research and production strategy. In reality this does not necessarily happen, neither in the educational nor the professional spheres. There are constant conceptual and formal inconsistencies, motivated by economic pressures, professional limitations and demands of all kinds, although fundamentally defined by a paradigmatic vision that defines the scope of design's research and execution. The following is a general characterization of the relationship that design research is estimated to have with the professional field, considering the realization of projects; the awareness of a method being used at the time of production and the registration process for the socialization of what is done. Emphasis is also placed on research description stemming from design sectors such as the academic and professional ones. Efforts are made to ponder design from a social perspective, since individuals are the fundamental reason of that which is planned and produced. When promptly thinking about culture and social order within design projects and research, it is possible to notice the inseparable influence of both aspects on strategic development, also determining the repercussions of its effectiveness. Three conceptual moments are addressed regarding design and research: their social condition; their external condition and their lack of substance. These moments are approached with sociological foundations as a starting point, in order to be consistent with the exhibition's objectives, seeking to raise awareness and revalue the social aspects of the processes when executing and researching from within the subject area, thus seeking to bring design into the realm of social and cultural debate by ratifying it as a dynamic profession result of a series of collective traits, which helps to shape thoughts.
\end{abstract}

Keywords: Design research - Social Design - Research - Design.

Resumo: Aparentemente é indiscutível que se impliquem aspectos sociais e culturais como parte da estratégia de pesquisa e produção de design, entretanto, isso é algo que não ocorre necessariamente, nem no âmbito formativo, nem no âmbito profissional. Constantemente é possível notar inconsistências conceituais e formais, motivadas por pressões econômicas, limitações profissionais e exigências de todo tipo, embora fundamentalmente definidas por una visão paradigmática que define os alcances da pesquisa e da realização do design. A seguir apresenta-se um panorama geral da relação que se acredita que faz parte da pesquisa em design com respeito ao campo profissional, considerando a realização de projetos; a conscientização de um método no momento de produzir e o processo de registro para divulgação do que se faz. Também se coloca ênfase na descrição da pesquisa sobre os setores do design gráfico no âmbito acadêmico, ademais do profissional. Procura-se abordar o design por uma perspectiva social, já que os indivíduos são a razão fundamental do que se projeta e produz. Ao pensar oportunamente a cultura e ordem social em projetos e pesquisas de design, observa-se a inseparável incidência de ambas em qualquer desenvolvimento estratégico, determinando-se também as repercussões de sua eficácia. São abordados três momentos conceituais respeito ao design e a pesquisa: sua condição social, sua condição externa e sua dessubstancialização. Esses momentos são abordados a partir de fundamentos sociológicos, com a finalidade de ser consistente com os objetivos 
da apresentação, ao procurar conscientizar e revalorizar os aspectos sociais dos processos do fazer e do pesquisar academicamente, e assim procurar colocar o design gráfico no debate social e cultural, ao ratifica-lo como profissão dinâmica que provem de aspectos coletivos, que contribui na configuração de pensamentos e incide em comportamentos.

Palavras chave: Pesquisa de design - design social - investigar - projetar.

[Las traducciones de los abstracts fueron supervisadas por el autor de cada artículo] 